\title{
Electromagnetic Modeling of the Propagation Characteristics of Satellite Communications Through Composite Precipitation Layers, Part II: Results of Computer Simulations
}

\author{
H.M. Al-Rizzo ${ }^{*}$, H.T. Al-Hafid ${ }^{* *}$, and J.M. Tranquilla ${ }^{* * *}$
}

${ }^{*}$ College of Information Science and Systems Engineering, University of Arkansas, 2801 South, University Avenue, Little Rock, AR 72204, and

Department of Electrical and Electronics Engineering, College of Engineering, Sultan Qaboos University, P.O. Box 33, Al Khod 123, Muscat, Sultanate of Oman; ${ }^{* *}$ Electrical Engineering Department, United Arab Emirates University, Al Ain, United Arab Emirates; ${ }^{* *} E M R$ Microwave Technology Corporation, 64 Alison Blvd., Suite 105, Fredericton, NB, Canada, E3C 1N2.

$$
\begin{aligned}
& \text { نمذجة انتشار الموجلت الكهرومغنلطيسية لمظومات الأقمار الصناعية خلللطبقات المطر } \\
& \text { المترلكمة : النتائج والمحلكلت المسابية }
\end{aligned}
$$

\section{مسبن ضافر الربزو ، حلغلطه الحلال ، وجم ترانكولا}

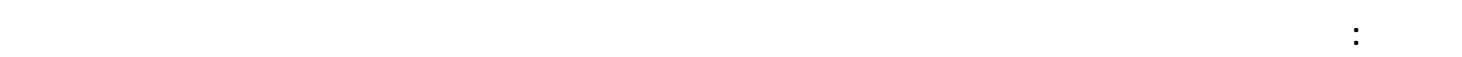

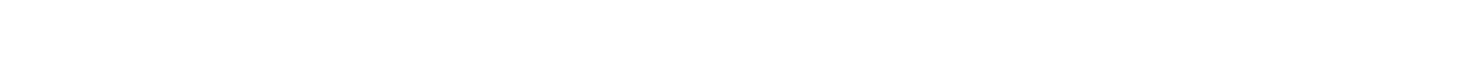

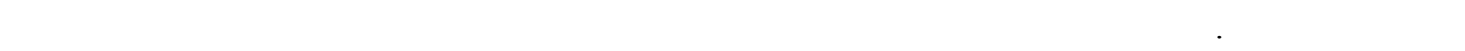

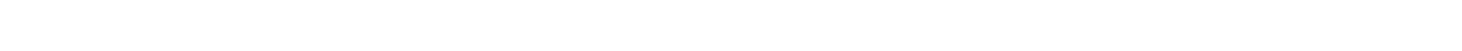
التوهين الناتج عن المطين الاعبار البهر.

\begin{abstract}
A versatile Propagation Simulation Program (PSP) is developed to assess the degrading effects caused by the concurrent occurrences of an arbitrary mixture of ice plates and needles, melting snow and raindrops which may impede the reliability of dual-polarized satellite communications systems carrying independent channels on a single radio path. Specifically, results are presented for the Cross Polarization Discrimination (XPD) due to ice and rain, differential attenuation, $\Delta \alpha$, and differential phase shift, $\Delta \phi$, due to rain and average specific attenuation, $\alpha$, and phase shift, $\phi$, due to the melting layer at hitherto unconsidered frequencies. The inclusion of an ice-cloud medium is found to possess significant effects on rain-induced XPD even for low ice concentrations, particularly at low fade levels. The relative contribution of the melting layer on rain-induced attenuation is extensively studied for frequencies from 1 to $100 \mathrm{GHz}$ and rain rates below $20 \mathrm{~mm} / \mathrm{h}$.
\end{abstract}

KEYWORDS : Dual-Polarized Satellite, Cross Polarization Discrimination .

A

versatile PSP has been constructed using the theoretical formulation established by Al-Rizzo, et al (1999) to run interactively on a 96 MB-RAM SunSparc 20 computer. The program evaluates the deleterious effects of the whole spectrum of precipitation existing in the lower part of the atmosphere on the performance of dual-polarized earth-to-space links operating at frequencies in the microwave and millimeter wave bands.

The input parameters to the program can be classified into two categories. First, link parameters which include frequency of operation, elevation angle, $\beta$, of the slant path as viewed from the earth station, and the 
polarization parameters of the dual-polarized transmitting, $\left(\gamma_{t 1}, \delta_{t 1}\right),\left(\gamma_{t 2}, \delta_{t 2}\right)$ and receiving, $\left(\gamma_{r 1}, \delta_{r 1}\right),\left(\gamma_{r 2}, \delta_{r 2}\right)$ antennas. For geo-stationary satellites employing linearly polarized transmission, the longitude, $\xi$, and latitude, $\eta$, of the earth terminal location are defined with respect to the sub-satellite point and its height above mean sea level.

For solid hydrometeors predominantly existing above the melting layer (Tranquilla and Al-Rizzo, 1994), the parameters include the fraction of plate-like crystals, $p$, the eccentricities of both plate-like and needle-like crystals, $e^{p, n}$, treated as oblate and prolate spheroidal Rayleigh scatterers, respectively. The canting angle parameters of the model are the mean $\chi_{0}$ and the standard deviation $\sigma_{\chi}$ of the normally distributed, transverse canting angle for both ice plates and needles in the incident polarization plane normal to the direction of propagation of the incident wave. For ice needles, $\psi_{0}, \sigma_{\psi}$ represent the relevant parameters of the normally distributed azimuthal rotation angle in the horizontal plane between the symmetry axis and the $x$-direction. Finally, the input parameters involve the vertical spatial extent, $H_{\mathcal{C}}$, and the average volume concentration of the ice cloud, $\langle V\rangle$, in units of $\mathrm{m}^{3}$ of ice per unit $\mathrm{m}^{3}$ of space.

Perhaps the most critical, but rarely known, parameter required in the estimation of the total raininduced attenuation experienced on a slant path is the spatial rain-rate profile along the propagation direction. Presently, three models are implemented in our program; a uniform, piecewise uniform (Persinger and Stutzman, 1980) and exponential (Stutzman and Dishman, 1982) spatial rain profile.

The parameters of the melting-layer model include the vertical depth of the bright band below the $0^{0} \mathrm{C}$ isotherm, temperature lapse rate, minimum and maximum radii of the eventually melted drops along with any prescribed particle-size spectrum function and the rain rate in $\mathrm{mm} / \mathrm{h}$. Based on the postulates of the thermodynamic model for the melting process (Al-Rizzo et al 2000), estimates of the density, fraction of the total mass of a melting snowflake, terminal fall speed, radius of the partially melted snowflake and the radius of the unmelted core, number of snowflakes of a given size per unit volume as well as the effective complex relative dielectric constant of the core region, corresponding to any given size of the eventually melted raindrop, are provided at any specified height downward steps in the melting zone until all of the snowflakes have been melted.

A close scrutiny of the literature revealed that the propagation of millimeter wave (MMW) signals from a satellite source to a ground-based receiver through composite atmospheric layers of precipitation received little attention so far. This dearth of published data stemmed primarily from the formidable numerical difficulties encountered in computing the scattering properties of an arbitrarily-shaped, 3-D scattering object whose size is comparable to or larger than the wavelength of the incident radiation. This is probably why most of the early (Oguchi, 1964, 1977), (Morrison and Cross, 1974), (Warner and Hizal, 1976), (Holt et al 1978) and more recent investigation (Ajase and Sadiku, 1995), (Li et al., 1995), (de Wolf and Zwiesler, 1996), (Seow et al. 1998) focused their efforts on frequencies less than $100 \mathrm{GHz}$. In contrast, the dispersive characteristics of $\Delta \alpha$ and $\Delta \phi$ are provided herein for rain rates from $2.54 \mathrm{~mm} / \mathrm{h}$ to 152.4 $\mathrm{mm} / \mathrm{h}$, raindrop temperatures of $0^{\circ} \mathrm{C}$ and $20^{\circ} \mathrm{C}$, and frequencies from 1 to $200 \mathrm{GHz}$. Results are presented for five raindrop size distributions (RSD), Laws-Parsons (LP) (Laws and Parsons, 1948), modified MarshallPalmer (MMP), drizzle (JD), thunderstorm (JT) (Olsen et al, 1978) and lognormal (LN) (Ajaya and Olsen, 1985). The most commonly accepted raindrop shapes are currently in use in our Generalized Multipole Technique software (GMT) (Al-Rizzo and Tranquilla, 1997). These include spherical, oblate spheroids with the axial ratio being dependent on the radius of the equivolume spherical drop (Oguchi, 1977), bimodal discrete distribution consisting of $P_{S}$ effective fraction of spherical and the remaining $\left(1-P_{S}\right)$ are oblate spheroids and the realistic shapes described by Pruppacher and Pitter (1971). In fact, there have been no treatments up to date that deal with the wide range of parameters involved in this paper.

\section{Results of Computer Simulations}

A versatile PSP has been developed based on the theoretical formulation developed by Al-Rizzo, et al (2000) in combination with the GMT single-particle scattering code (Al-Rizzo and Tranquilla, 1997). Reliable estimates of $\alpha, \phi$ and XPD due to isolated ice clouds, melting snow and rain as well as the overall degradation caused by the concurrent occurrences of various storm activities including mixed ice/rain and a segmented melting snow/rain events are provided in this paper. 


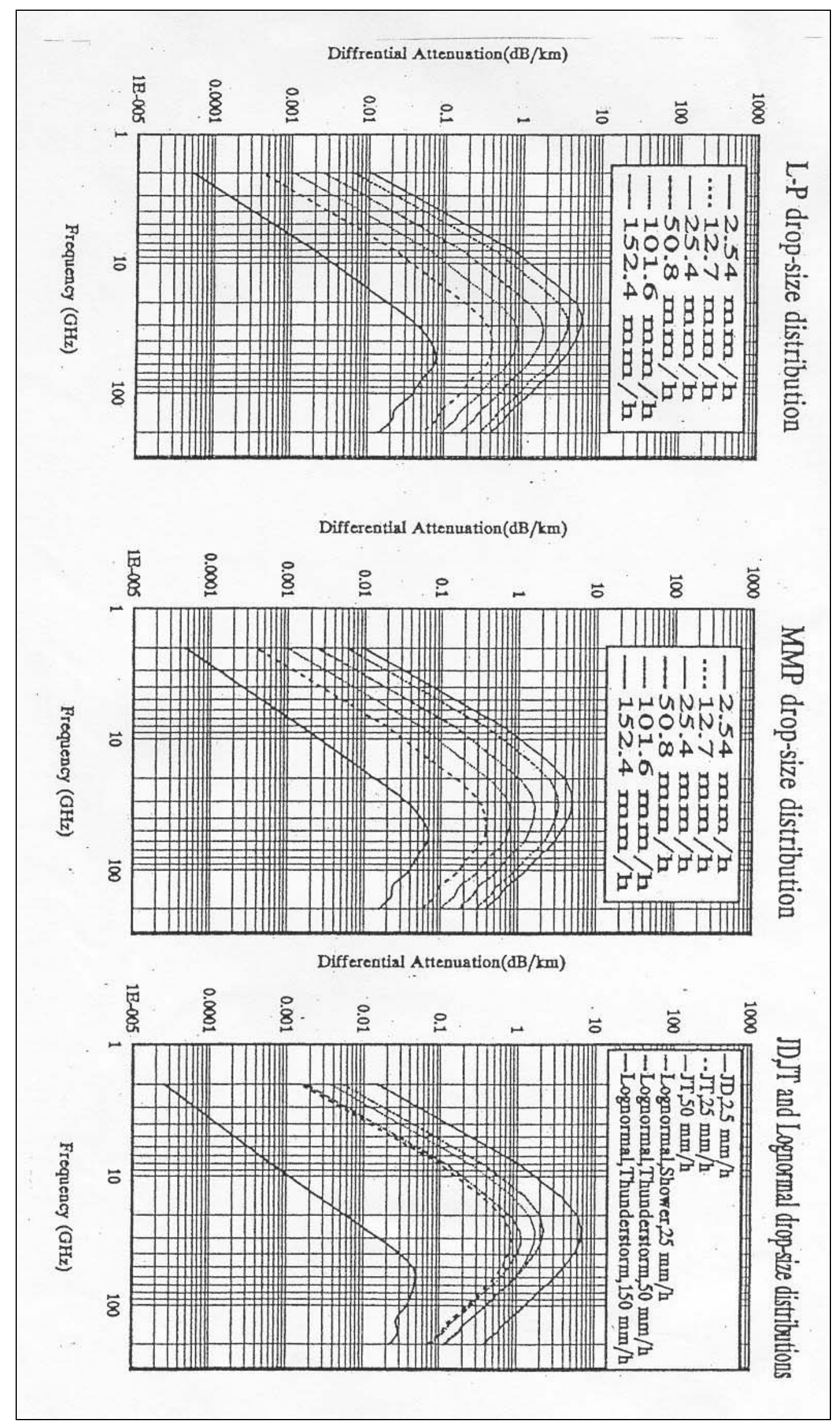

Figure 1A. Frequency characteristics of rain-induced $\Delta \alpha$ (Horizontal-Vertical) over a range of rain rates and for several RSD models, P-P drops at $20^{\circ} \mathrm{C}$. 
AL-RIZZO, AL-HAFID AND TRANQUILLA

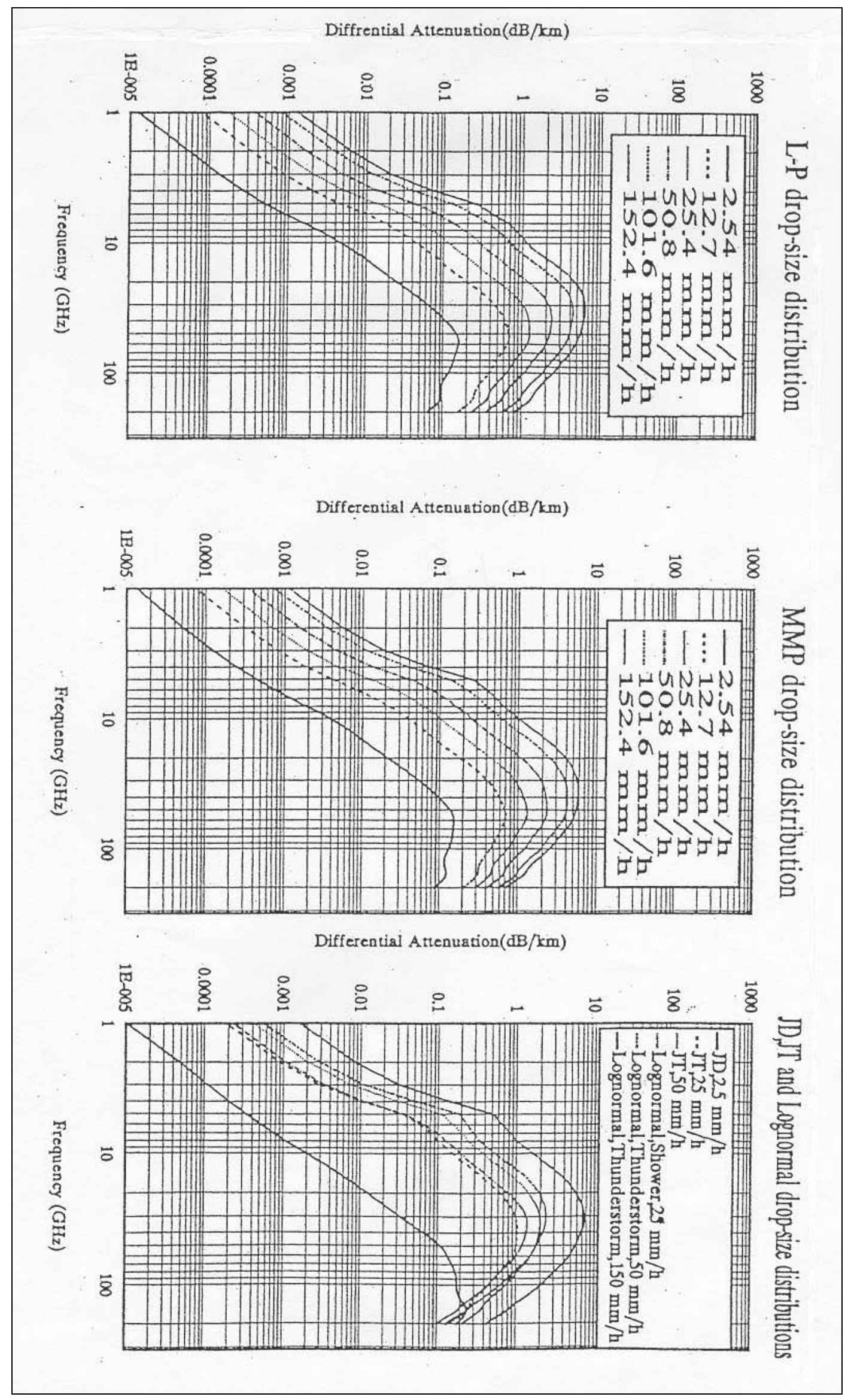

Figure 1B. Frequency characteristics of rain-induced $\Delta \alpha$ (Horizontal-Vertical) over a range of rain rates and for several RSD models, P-P drops at $0^{\circ} \mathrm{C}$. 


\section{MODELING THE PROPAGATION OF SATELLITE COMMUNICATIONS}

Figures 1 and 2 present the dispersive characteristics of $\Delta \alpha$ and $\Delta \phi$ experienced by a $1-\mathrm{km}$ long, uniformly filled rain path for rain rates from 2.54 to $152.4 \mathrm{~mm} / \mathrm{h}$. Equi-oriented P-P drops with their symmetry axes oriented along the vertical direction are considered (results for oblate drops are available upon request from the authors).

The results are given for LP (Laws and Parsons, 1948), MMP, JT, JD (Olsen, Rogers, and Hodge, 1978) and LN RSD (Ajaya and Olsen, 1985), drop temperatures of $0^{\circ} \mathrm{C}$ and $20^{\circ} \mathrm{C}$ and frequencies from 1 to $200 \mathrm{GHz}$ with an increment of $1 \mathrm{GHz}$. With the exception of the limited data presented by Oguchi (1977), Fang and Lee (1978), and Li et al. (1995) previous results for $\Delta \alpha$ and $\Delta \phi$ were restricted to only oblate drops (Oguchi, 1964), (Morrison and Cross, 1974), (Warner and Hizal, 1976), (Holt, Uzunoglu, and Evans, 1978), (Seow et al. 1998) at a single drop temperature and for frequencies less than $100 \mathrm{GHz}$ using much wider frequency increments. It should be mentioned that the perturbation approximation utilized by Oguchi (1964) and Li et al. (1995) yields less accurate results, particularly for $\Delta \phi$ above $30 \mathrm{GHz}$.

It is evident from Figures 1 and 2 that $\Delta \phi$ dominate rain-induced XPD below $10 \mathrm{GHz}$ whereas $\Delta \alpha$ is negligibly small. The differential phase shift $\Delta \phi$ peaks at a frequency in the range from 17 to $24 \mathrm{GHz}$ and then falls off rapidly changing into positive values in the MMW region. The differential attenuation increases rapidly with frequency and reaches its maximum at a frequency, which depends on rain intensity and then levels off, and decreases at higher MMW frequencies due to the contribution of the less-deformed smaller drops, which dominate the cause of attenuation at shorter wavelengths. There is little difference in the results for the various RSD below $10 \mathrm{GHz}$.

Rain-induced XPD versus $\alpha$ is next examined in Figure 3 where it is well known that a simple empirical relationship, insensitive to space-profile of rain, exists between their measured statistics for both terrestrial and satellite-earth paths (Allnutt, 1989).

The calculations are performed at 50,80,110,140,170 and $200 \mathrm{GHz}$ for a $12.7 \mathrm{~mm} / \mathrm{h}$ uniform rain structure, given perfectly polarized and exactly aligned antennas. The variation of $\alpha$ has been affected by changing the extent of rain volume along the propagation direction. The results show that significant depolarization should be expected at such MMW frequencies even for a low rainfall rate of $12.7 \mathrm{~mm} / \mathrm{h}$ and for fade levels that could possibly occur along distances greater than $5 \mathrm{~km}$. On the other hand, XPD at a given $\alpha$ increases with frequency because smaller raindrops of less eccentricity, while making little contribution to depolarization, contribute heavily to rain-induced attenuation owing to their high extinction cross section. Also, the difference between the XPD encountered by horizontally and vertically polarized transmission is negligibly small.

In Figure $4 \alpha$, XPD and relative cross-polar phase are shown at $30 \mathrm{GHz}$ for a $5-\mathrm{km}$ slant path of $\beta=$ $45^{0}$ as a function of rainfall rate from 1 to $160 \mathrm{~mm} / \mathrm{h}$ utilizing a uniform, quasi-uniform (Persinger and Stutzman, 1980) and exponential spatial rain-rate distribution (Stutzman and Dishman, 1982) (results at 12 and $24 \mathrm{GHz}$ are available upon request from the authors). The relative cross-polar phase is an important parameter when inferring rain anisotropy and is also used for canceling cross talk in one channel of a dualpolarized system by inserting a properly leveled and phase shifted sample of the signal received in the orthogonal polarized channel. The results emphasize the dominant role of the rain spatial structure, particularly on total rain-induced attenuation. Raindrop shape and orientation constitute the major influence on XPD as well as the phase delay encountered separately by the co- and cross-polar signals. Attenuation for horizontally polarized transmission is greater than for vertical polarization while the XPD for vertical polarization is in general greater than for horizontal transmission. The XPD is relatively insensitive to the RSD function. Since the deformation of oblate and/or P-P drops increases with the drop size, XPD is lower at higher rain rates due to the greater number of large-sized drops.

Figure 5 depicts $\alpha$ and $\phi$ at $30 \mathrm{GHz}$ versus depth from the $0^{\circ} \mathrm{C}$ isotherm in descending steps of $25 \mathrm{~m}$ for a plane wave vertically incident on the upper boundary of a melting layer. The results are shown for the MP and LP RSD corresponding to rain rates of $2.5,5,7.5,10,12.5$ and $15 \mathrm{~mm} / \mathrm{h}$ assuming a $6^{\circ} \mathrm{C}$ linear lapse rate of temperature (results at 12 and $24 \mathrm{GHz}$ are available upon request from the authors). It can be seen that $\alpha$ for the initially frozen snowflakes remains low for all rain rates considered. However, at complete melting their values become significant, particularly for the higher rain rates. At a given height, $\alpha$ and $\phi$ increase with frequency and rain rate. The height profiles reach their peak values at around $210 \mathrm{~m}$ below the $0^{0} \mathrm{C}$ for all rain rates considered. Depending on the rain intensity, the ratio of peak $\alpha$ to its value upon complete melting can be in excess of 3 .

The average specific attenuation and phase delay are next obtained by numerically integrating the vertical profiles of $\alpha$ and $\phi$ starting from the $0^{\circ} \mathrm{C}$ isotherm along the signal path within the melting region and then normalizing the results by the depth of the layer (Kharadly and Owen, 1988). We have performed 


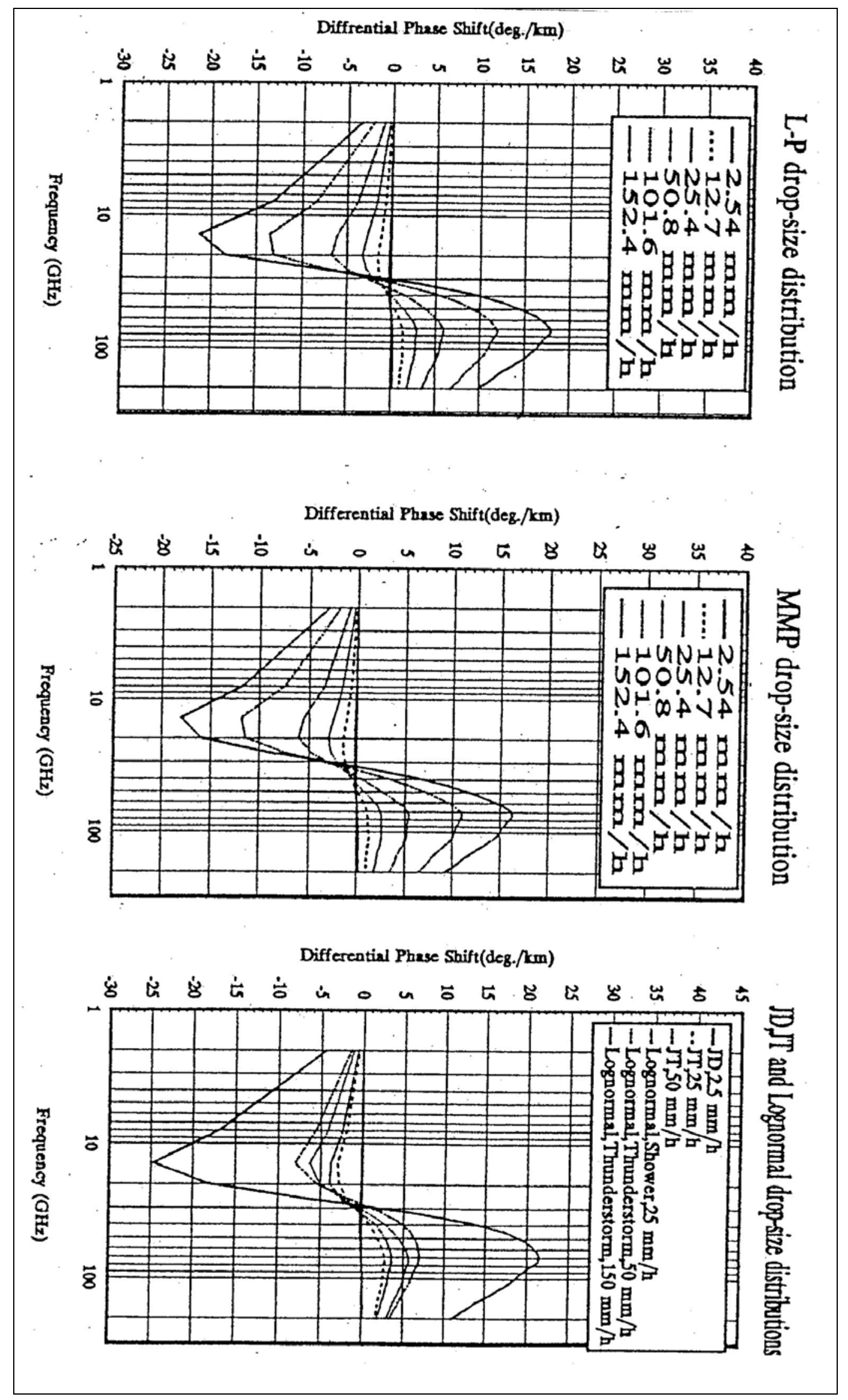

Figure 2A. Frequency characteristics of rain-induced $\Delta \phi$ (Horizontal-Vertical) over a range of rain rates and for several RSD models, P-P drops at $20^{\circ} \mathrm{C}$. 


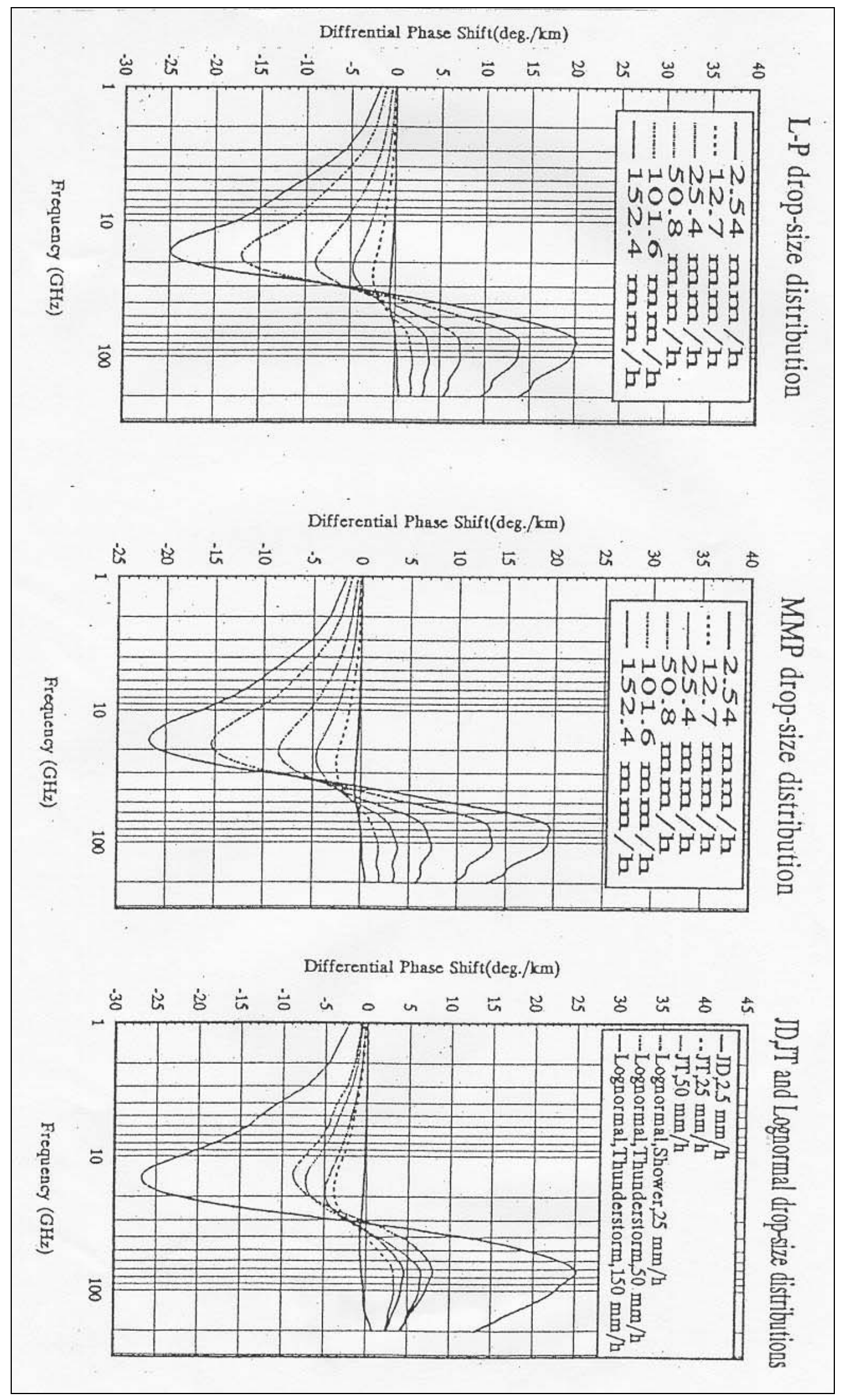

Figure 2B. Frequency characteristics of rain-induced $\Delta \phi$ (Horizontal-Vertical) over a range of rain rates and for several RSD models, P-P drops at $0^{0}$ 
AL-RIZZO, AL-HAFID AND TRANQUILLA

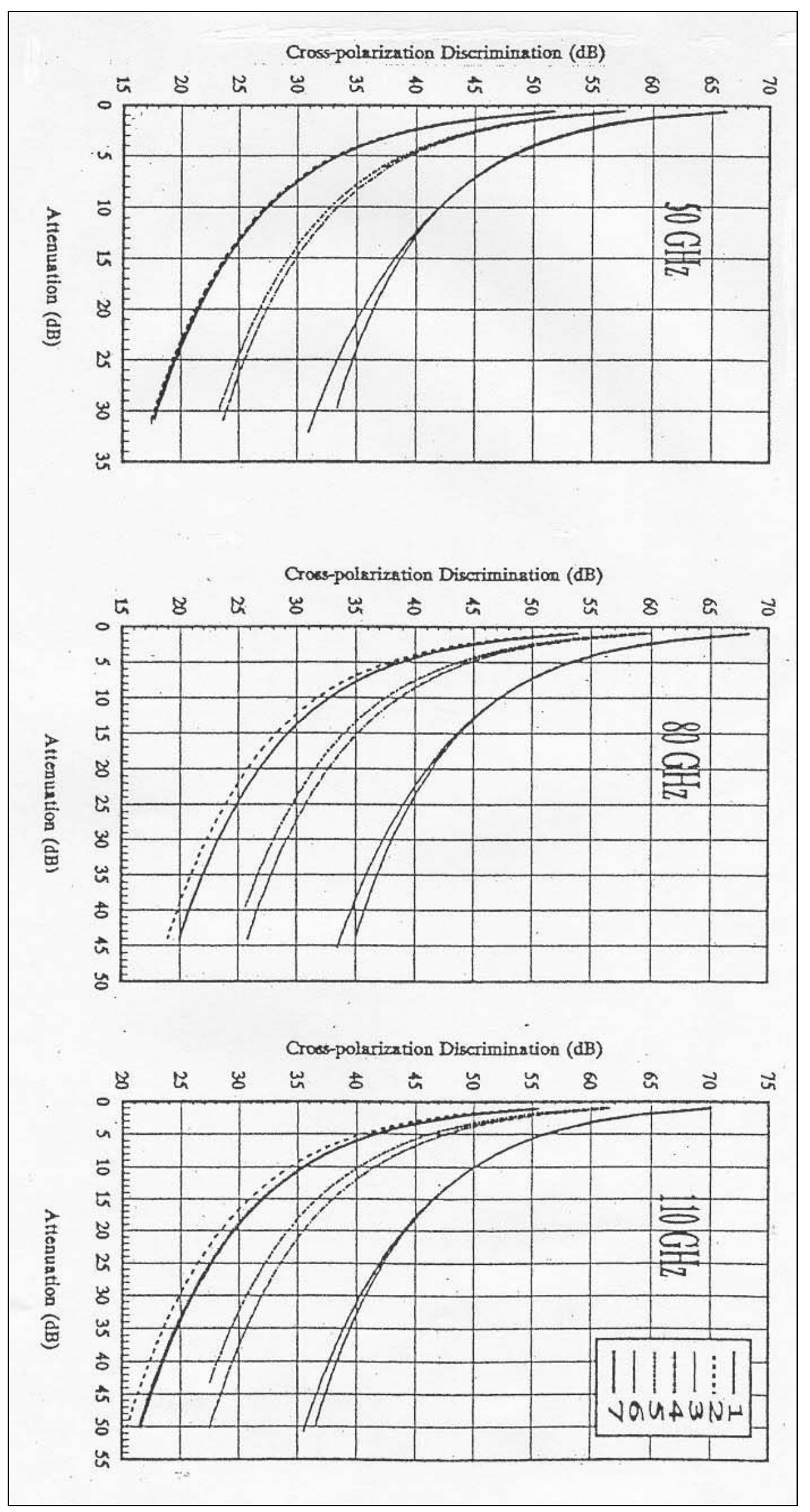

Figure 3. See next page for caption. 


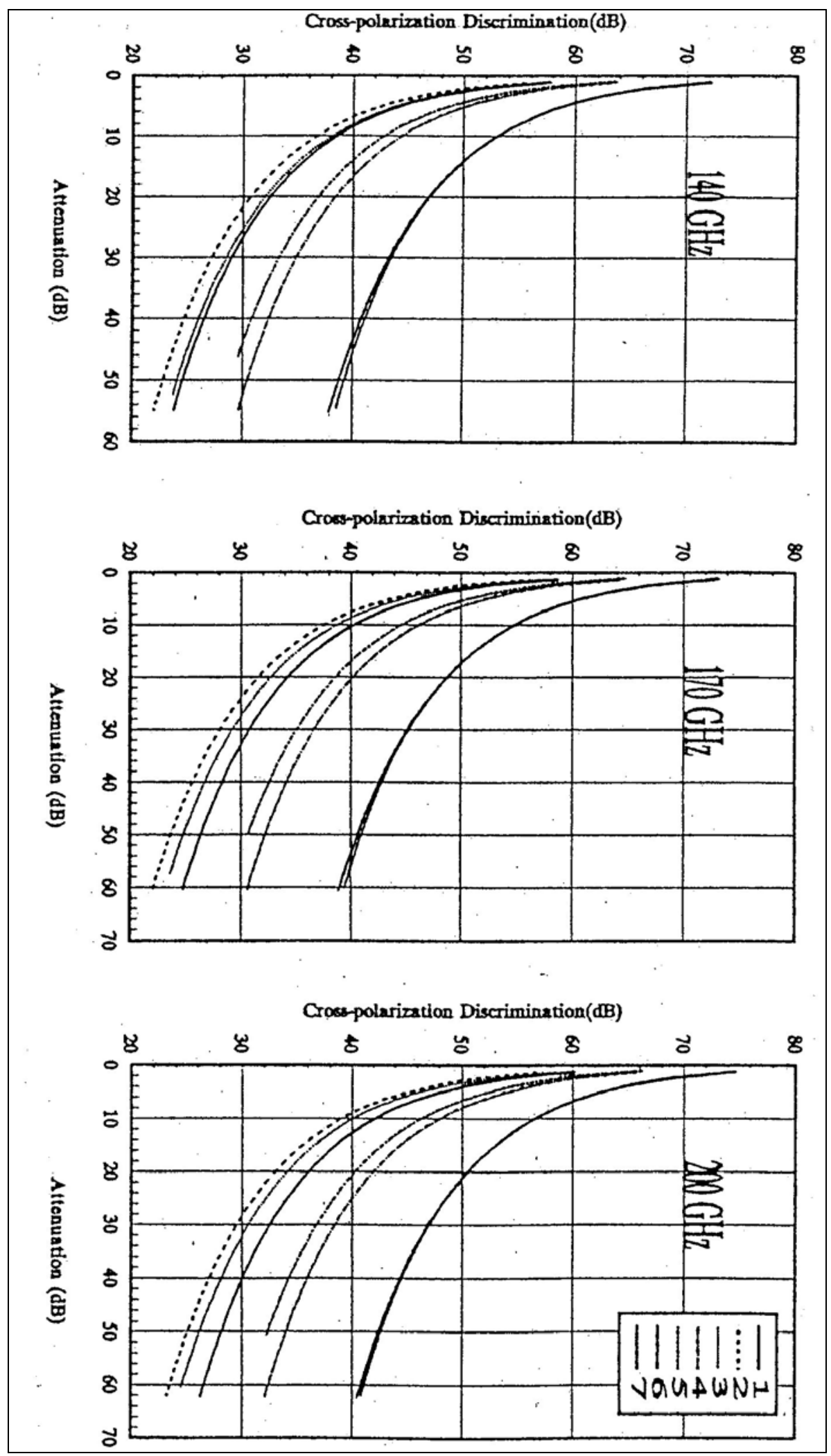

Figure 3. Rain-induced XPD versus $\alpha$ for a rain rate of $12.7 \mathrm{~mm} / \mathrm{h}$.

(1) RHCP, P-P drops at $20^{\circ} \mathrm{C}$, MMP RSD, $\theta_{0}=0^{0}, \sigma_{\theta}=12^{0}$.

(2) $60 \%$ oblate, $40 \%$ spherical drops, remaining parameters same as in (1)

(3) $0^{0} \mathrm{C}$ drop temperature, remaining parameters same as in (2)

(4) $\theta_{0}=5^{0}, \sigma_{\theta}=30^{\circ}$, remaining parameters same as in (1)

(5) LP RSD, remaining parameters same as in (4)

(6) Horizontal polarization, $\theta_{0}=5^{0}, \sigma_{\theta}=30^{0}$, remaining parameters same as in (1)

(7) Vertical polarization, remaining parameters same as in (1) 


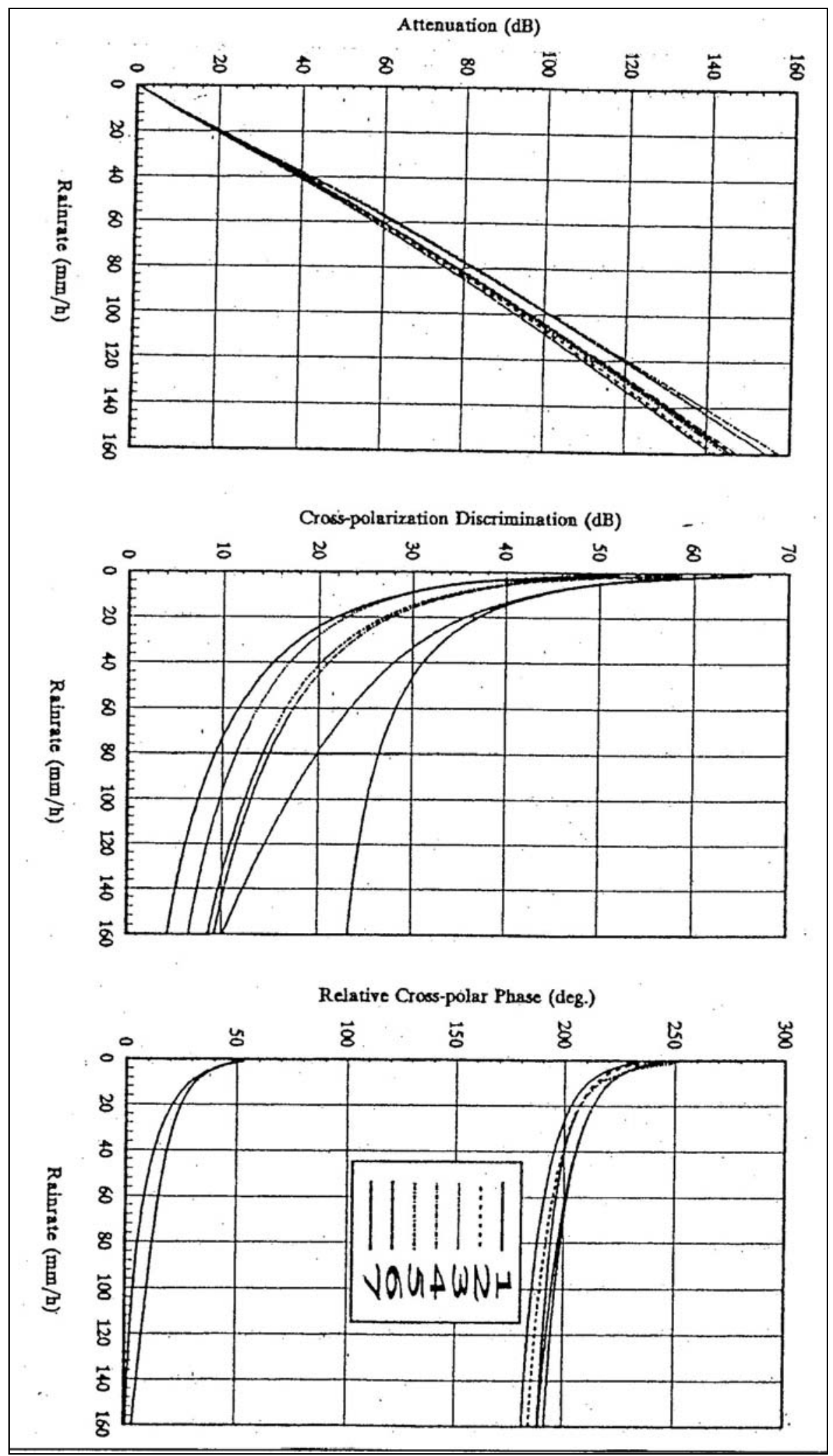

Figure 4A. Rain-induced $\alpha$, XPD, and relative cross-polar phase along a satellite-earth path of $\beta=45^{\circ}$ at 30 $\mathrm{GHz}$ versus rainfall rate for a uniform rain-rate spatial distribution, 5-km propagation path.
(1) RHCP, P-P drops at $0^{\circ} \mathrm{C}$, MMP RSD, $\theta_{0}=0^{0}, \sigma_{\theta}=12^{0}$.
(2) $20^{\circ} \mathrm{C}$ drop temperature, remaining parameters same as in (1)
(3) $60 \%$ oblate, $40 \%$ spherical drops, remaining parameters same as in (1)
(4) $\theta_{0}=5^{0}, \sigma_{\theta}=30^{0}$, remaining parameters same as in (1)
(5) LP RSD, remaining parameters same as in (4)
(6) Horizontal polarization, $\theta_{0}=5^{0}$, constant canting angle model, remaining parameters same as in (1)
(7) Vertical polarization, remaining parameters same as in (6) 


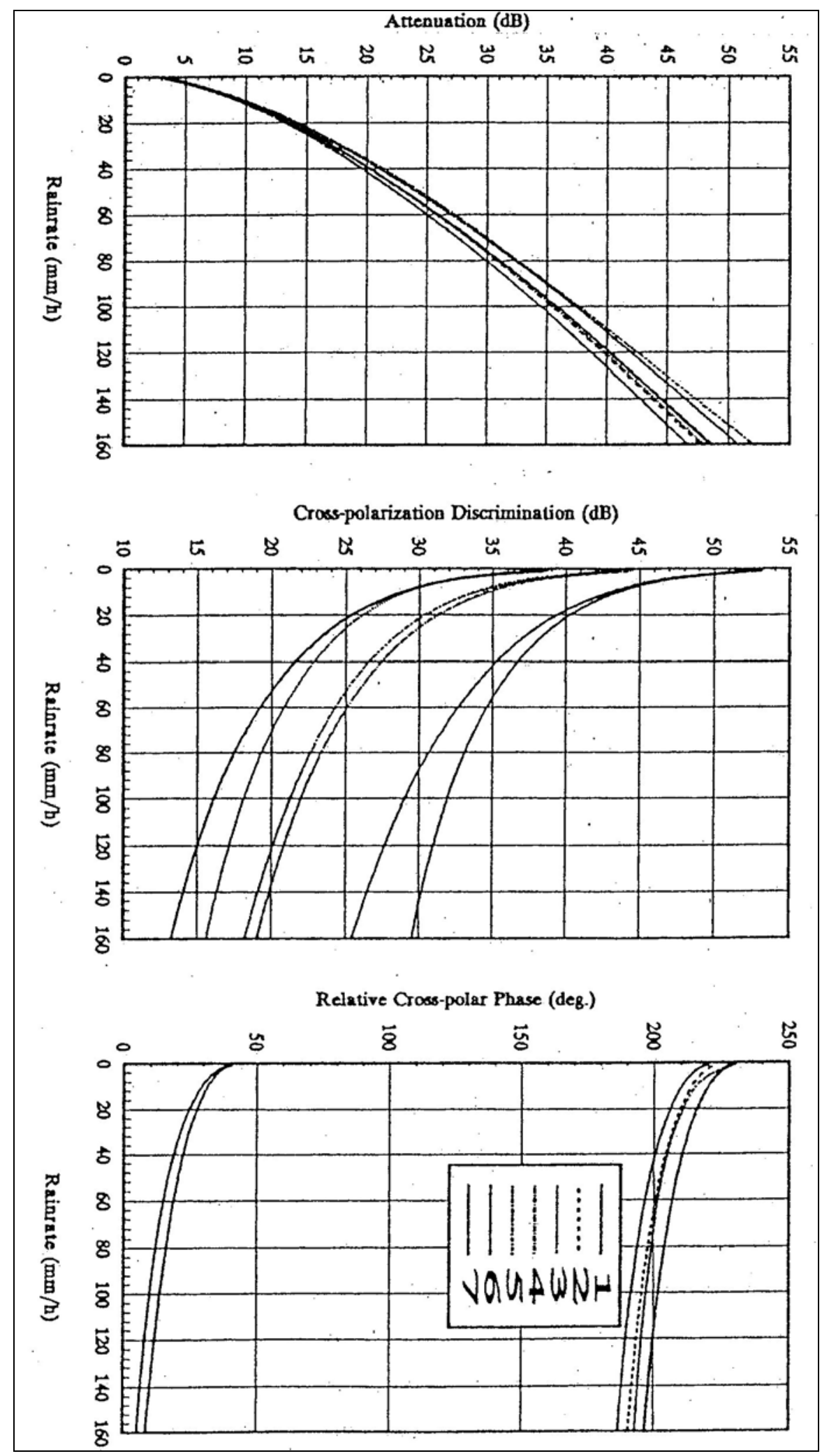

Figure 4B. Rain-induced $\alpha$, XPD, and relative cross-polar phase along a satellite-earth path of $\beta=45^{\circ}$ at $30 \mathrm{GHz}$ versus rainfall rate for a quasi-uniform rain-rate spatial distribution, $5-\mathrm{km}$ propagation path.

(1) RHCP, P-P drops at $0^{\circ} \mathrm{C}$, MMP RSD, $\theta_{0}=0^{0}, \sigma_{\theta}=12^{0}$.

(2) $20{ }^{\circ} \mathrm{C}$ drop temperature, remaining parameters same as in (1)

(3) $60 \%$ oblate, $40 \%$ spherical drops, remaining parameters same as in (1)

(4) $\theta_{0}=5^{0}, \sigma_{\theta}=30^{0}$, remaining parameters same as in (1)

(5) LP RSD, remaining parameters same as in (4)

(6) Horizontal polarization, $\theta_{0}=5^{0}$, constant canting angle model, remaining parameters same as in (1)

(7) Vertical polarization, remaining parameters same as in (6) 


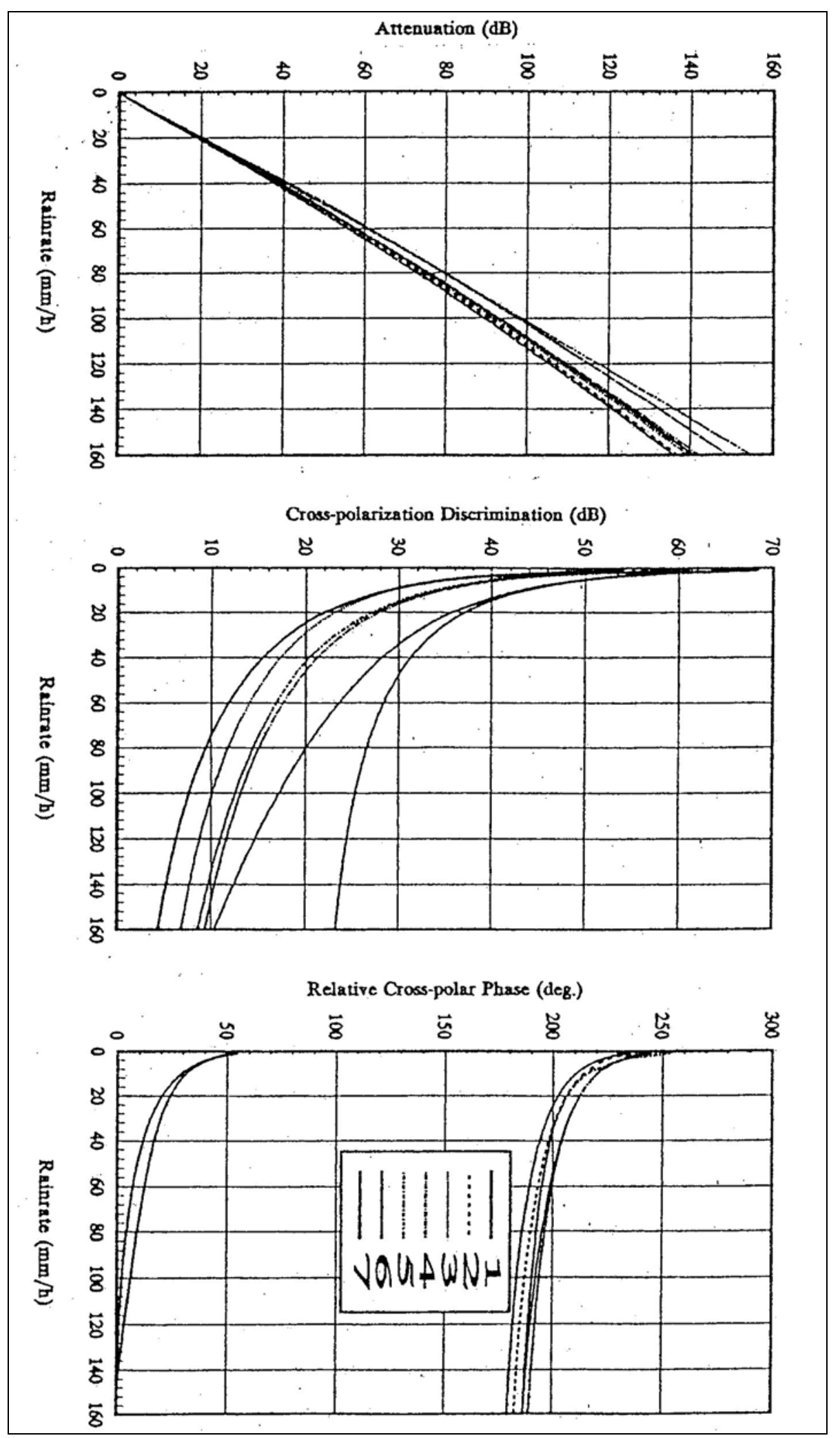

Figure 4C. Rain-induced $\alpha$, XPD, and relative cross-polar phase along a satellite-earth path of $\beta=45^{\circ}$ at 30 $\mathrm{GHz}$ versus rainfall rate for an exponential rain-rate spatial distribution, 5-km propagation path.
(1) RHCP, P-P drops at $0^{\circ} \mathrm{C}$, MMP RSD, $\theta_{0}=0^{0}, \sigma_{\theta}=12^{0}$.
(2) $20^{\circ} \mathrm{C}$ drop temperature, remaining parameters same as in (1)
(3) $60 \%$ oblate, $40 \%$ spherical drops, remaining parameters same as in (1)
(4) $\theta_{0}=5^{0}, \sigma_{\theta}=30^{0}$, remaining parameters same as in (1)
(5) LP RSD, remaining parameters same as in (4)
(6) Horizontal polarization, $\theta_{0}=5^{0}$, constant canting-angle model, remaining parameters same as in (1)
(7) Vertical polarization, remaining parameters same as in (6) 


\section{MODELING THE PROPAGATION OF SATELLITE COMMUNICATIONS}

extensive computations for frequencies from 1 to $100 \mathrm{GHz}$ using the MP and LP RSD for the underlying rain, the results of which are given in Figures 6 and 7.

Figure 6A also depicts the microwave attenuation obtained from the empirical model of Dissanayake and McEwan (1978) which is valid for frequencies from $9 \mathrm{GHz}$ to $30 \mathrm{GHz}$ under the assumption of MP RSD.

Also, in Figures 6B and 7B results obtained from Kharadly and Owen (1988) are shown using the LP RSD where the ratio of the melted volume to the total volume of the snow particle is assumed to be linearly proportional to the depth within the melting region.

The simulations seem to compare reasonably well although the vertical profiles of $\alpha$ and $\phi$ reported by Kharadly and Owen (1988) were larger than those obtained by our model with their peaks shifted towards smaller heights.

However, the average values of $\alpha$ and $\phi$ are still in favorable agreement except for $\phi$ above $40 \mathrm{GHz}$ where it appears that there are significant differences which may be attributed to the improper modeling of the forward scattering parameters.

This problem has been avoided in our model by employing an exact solution to the canonical problem of EM scattering from concentric layered snowflakes. Klassen (1988) also pointed out that the specific attenuation in the upper parts of the melting layer as reported by Kharadly and Owen (1988) were much larger than those obtained by his model.

It should be noted that Evans and Holt (1980) presented a theoretical formulation for propagation through composite layers of ice clouds and rain where disk- and needle-like crystals were modeled by oblate and prolate spheroids, respectively, both with unity eccentricity.

The improved ice-cloud model implemented in our PSP (Tranquilla and Al-Rizzo, 1994) extends its applicability range to the general case of a bimodal distribution of oblate and prolate spheroids with any specified eccentricity.

In Figure 8 the XPD on a slant down-path, Right Hand Circularly Polarized (RHCP) transmission of $\beta$ $=45^{0}$ is displayed against $\psi_{0}$ for a layered ice/rain medium. An ice cloud, $H_{C}=1 \mathrm{~km},\langle V\rangle=10^{-7} \mathrm{~m}^{3} / \mathrm{m}^{3}$ is segmented with a $10 \mathrm{~mm} / \mathrm{h}$ uniform rain medium, comprising P-P drops and a total path length of $3 \mathrm{~km}$ is assumed. Also shown are cases pertaining to ice-only events with $p=0$ and 0.5 .

The overall transmission matrix is obtained by multiplying the individual matrices of the ice and rain media and ensuring the continuity of the polarization directions of the fields exiting the ice cloud and impinging on the rain segment. The rain introduces a total attenuation of $0.7695,3.5853$ and $5.6865 \mathrm{~dB}$ whereas the rain-induced XPD levels are 41,35 , and $33 \mathrm{~dB}$ for the 12, 24, and $30 \mathrm{GHz}$ frequencies, respectively.

At a given rain rate, the interaction of the two media could have a significant effect even at low ice concentrations, particularly when the symmetry axis of the ice needles is perpendicular to the direction of propagation.

The rain medium has the effects of damping the excursions and shifting the position of minimum depolarization, originally caused by the change in the orientation of ice needles.

The XPD versus $\alpha$ is shown in Figure 9 for a RHCP signal traversing a rain and a mixed ice/rain medium along a slant path of $\beta=45^{\circ}$. The ice crystals are uniformly distributed within a cloud of $H_{C}=1$ $\mathrm{km},\langle V\rangle=10^{-7} \mathrm{~m}^{3} / \mathrm{m}^{3}$. The variation of $\alpha$ is accomplished by changing the rainfall rate from 1 to $50 \mathrm{~mm} / \mathrm{h}$, in steps of $1 \mathrm{~mm} / \mathrm{h}$. A uniform rain-rate distribution is assumed for a $5-\mathrm{km}$ slant path through the rain segment.

The results show that the ice-cloud medium contributes significantly to depolarization, particularly at low fade levels. At a given $\alpha$ and the three fractions of ice plates considered, $p=0,0.5$ and 1 , the XPD increases in that order. The rain-induced depolarization, however, dominates the overall XPD at the higher attenuation levels.

For satellite-earth links operating above $10 \mathrm{GHz}$, rain attenuation appears to be the dominant cause of propagation impairment.

However, scattering and absorption introduced by an ensemble of inhomogeneous snowflakes in the melting layer must be accounted for to accurately predict the total induced attenuation (Dissanayake and McEwan, 1978), (Kharadly and Owen, 1988), (Klaassen, 1988).

Figure 10 depicts the attenuation resulting from a model consisting partly of rain mixed with melting snow particles as well as for rain without a melting layer, drawn as a function of rainfall rate. The MP and LP RSD are employed for a RHCP transmission, $\beta=45^{\circ}$ and for frequencies of 12,24 and $30 \mathrm{GHz}$. 
The raindrops are assumed at $0^{\circ} \mathrm{C}$ and to have the P-P shapes. Furthermore, the path length through the rain region is taken as $5 \mathrm{~km}$ with a uniform rain-rate distribution. A Gaussian canting angle distribution is assumed with mean canting angle, $\theta_{0}=0^{0}$ and standard deviation $\sigma_{\theta}=12^{0}$. The total path length through the melting layer is fixed at $0.5 \mathrm{~km}$.

The computations are made for rain rates up to $20 \mathrm{~mm} / \mathrm{h}$, consistent with those encountered in stratiform precipitation. For all rain rates and frequencies considered, the relative importance of the meltinglayer attenuation is significant although the rain-induced attenuation is dominant due to the longer path inside the rainy portion of the total slant path.

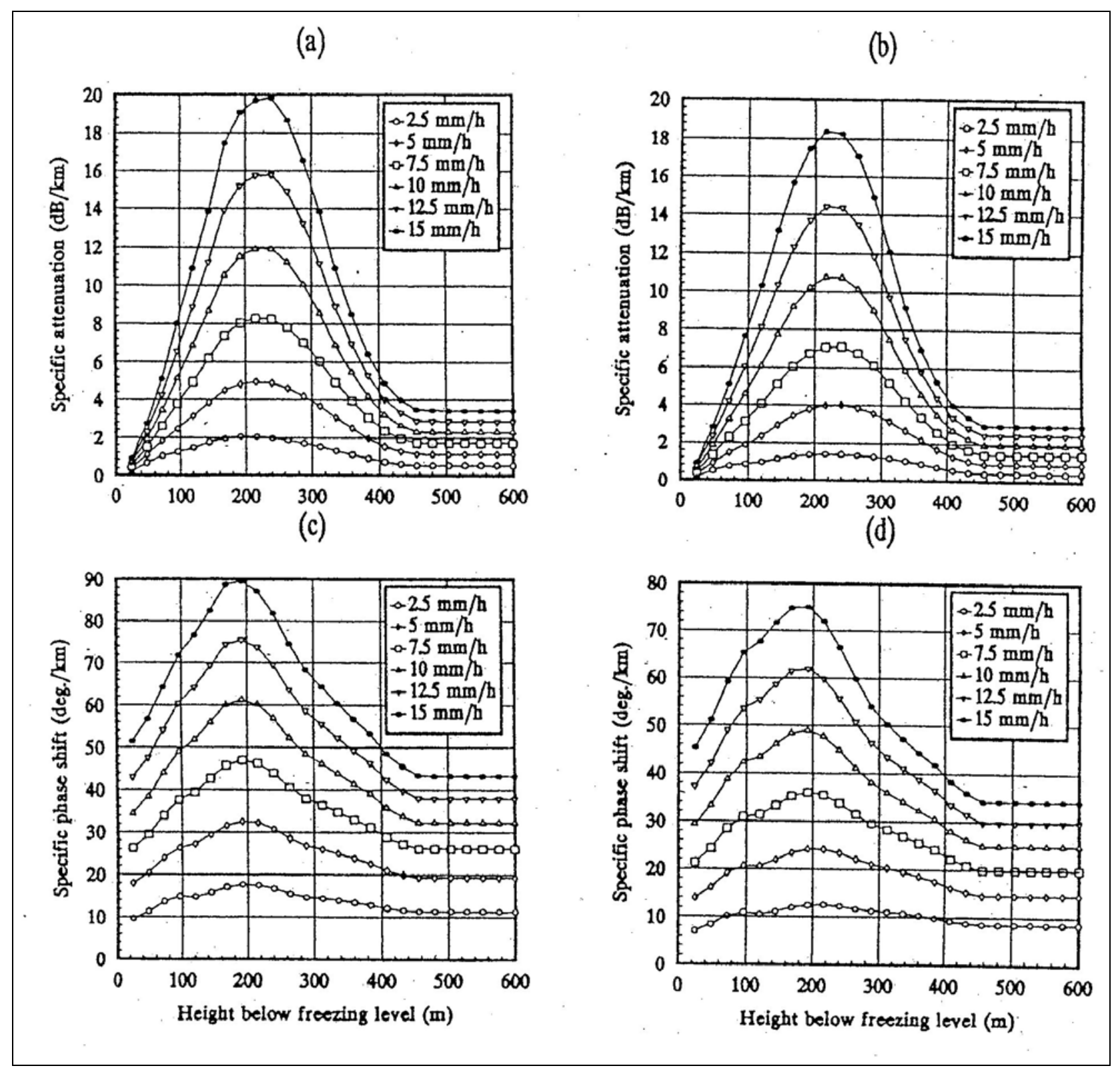

Figure 5. Vertical profiles of $\alpha$ and $\phi$ in the melting layer. (a),(c) $30 \mathrm{GHz}$, MP RSD (b),(d) $30 \mathrm{GHz}$, LP RSD 


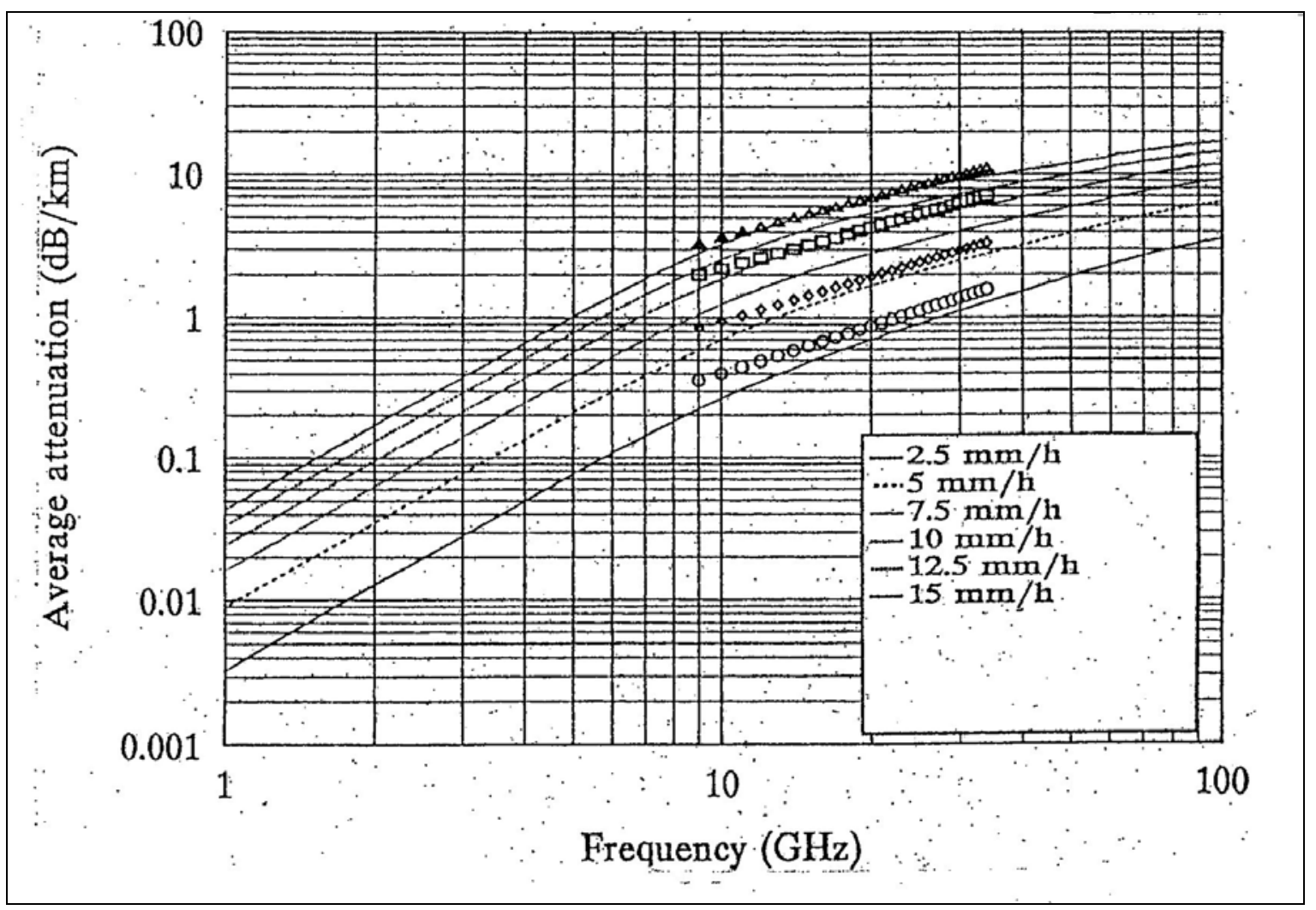

Figure 6A. Average attenuation properties of the melting layer as a function of frequency, MP RSD (o $2.5 \mathrm{~mm} / \mathrm{h}$, $\diamond 5 \mathrm{~mm} / \mathrm{h}, \square 10 \mathrm{~mm} / \mathrm{h}, \triangle 15 \mathrm{~mm} / \mathrm{h}$, Dissanayake and McEwan, 1978).

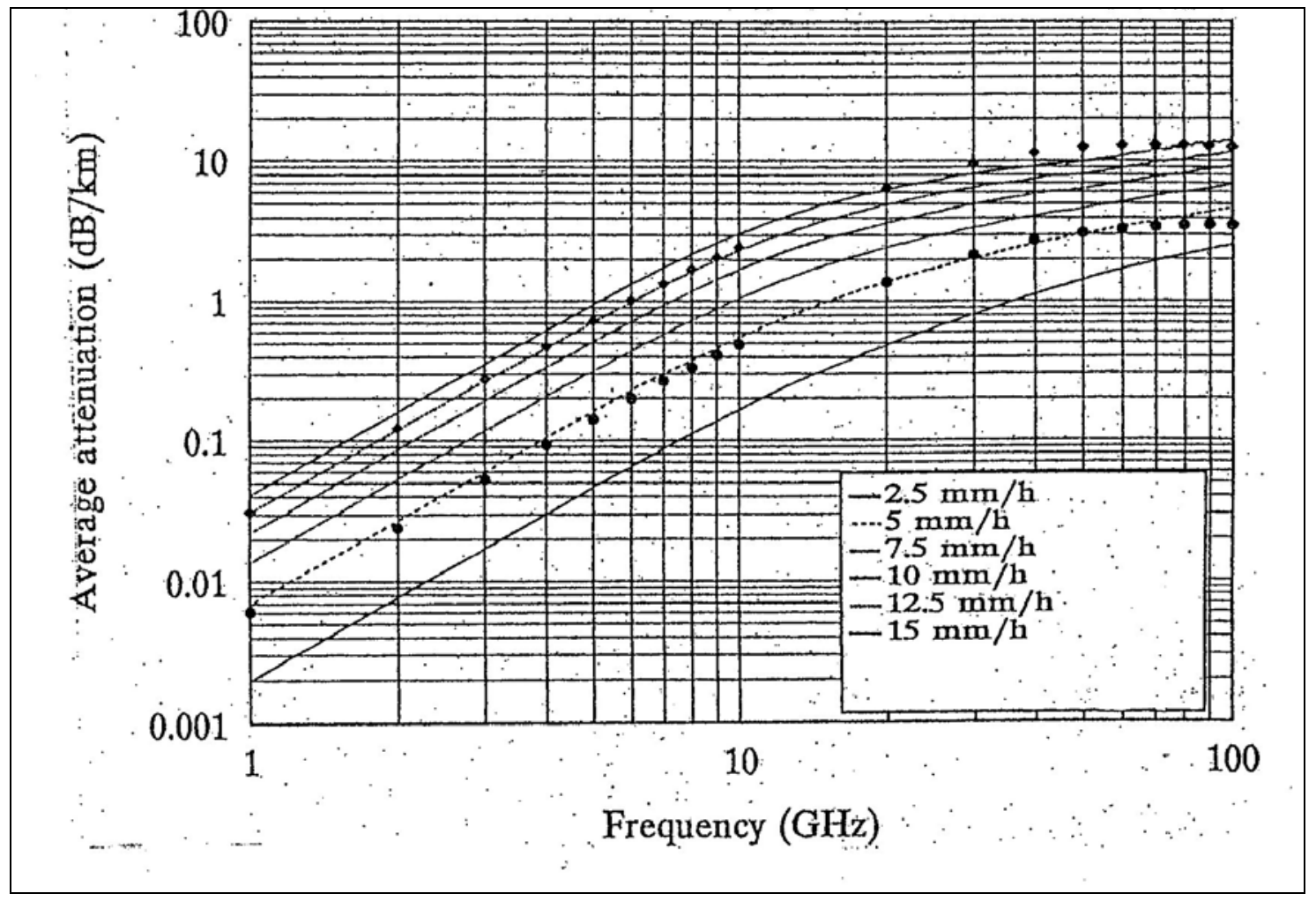

Figure 6B. Average attenuation properties of the melting layer as a function of frequency, LP RSD ( $5 \mathrm{~mm} / \mathrm{h}$, - $12.07 \mathrm{~mm} / \mathrm{h}$, Kharadly and Owen, 1988). 


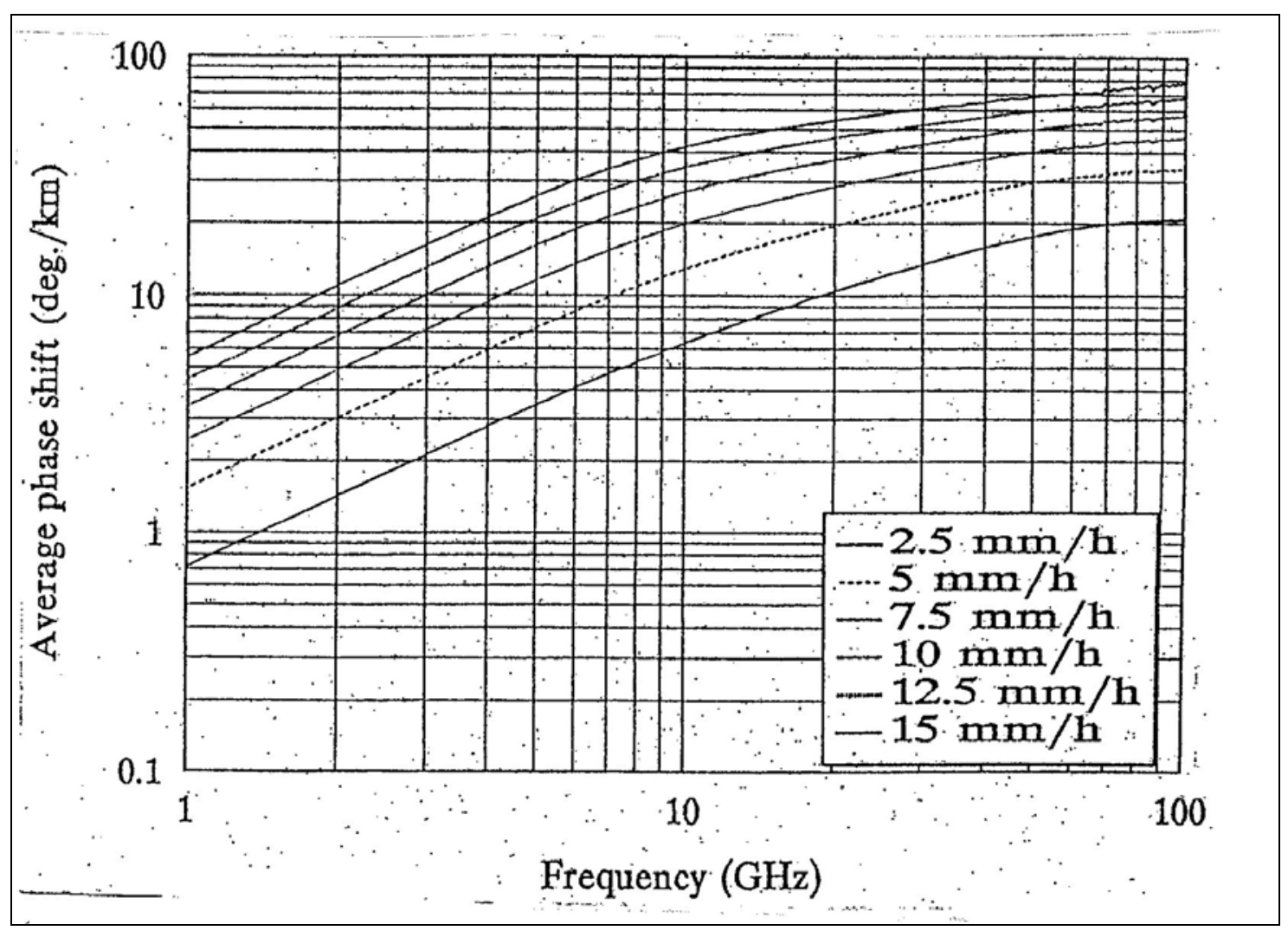

Figure 7A. Average phase shift properties of the melting layer as a function of frequency, MP RSD.

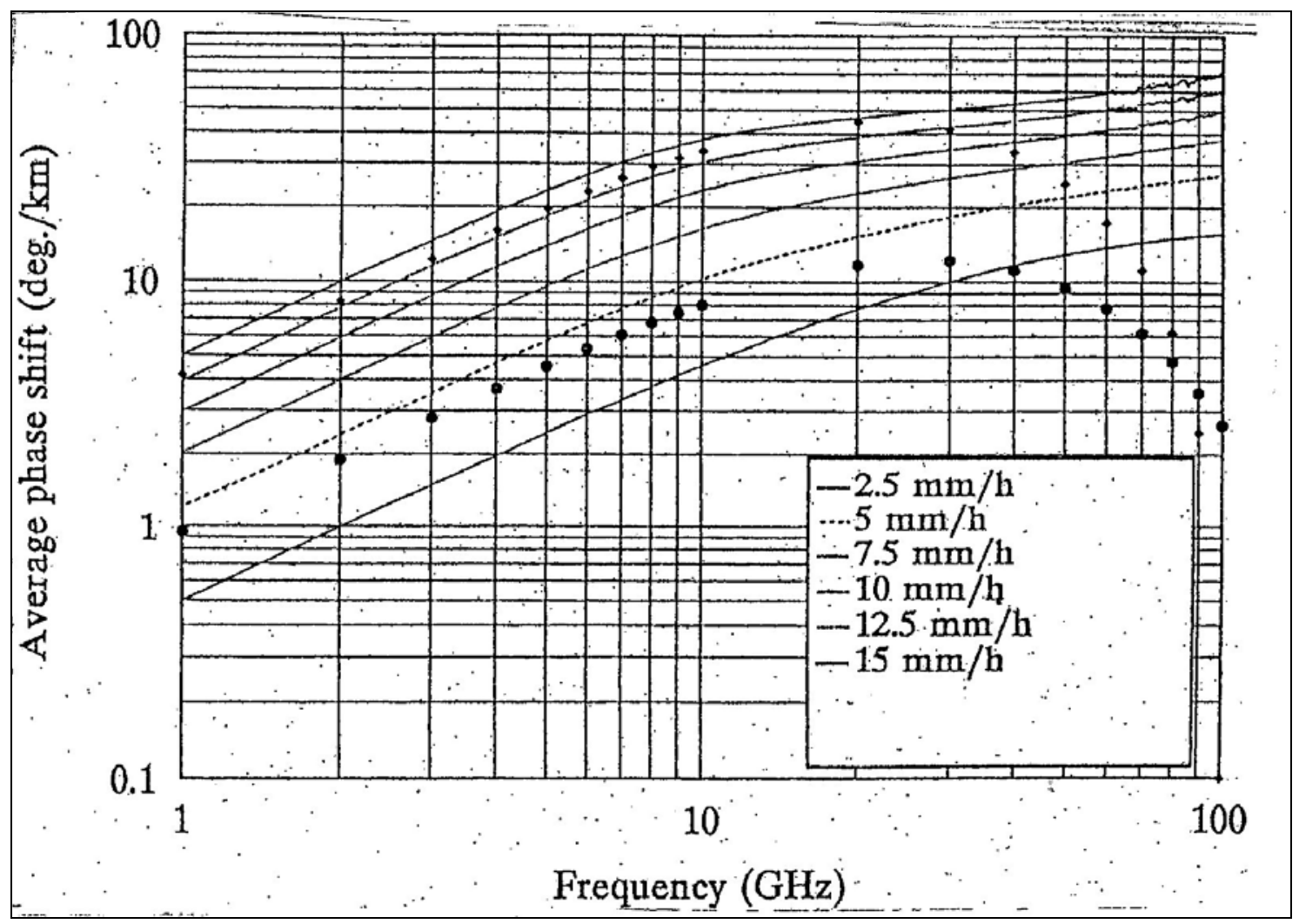

Figure 7B. Average phase shift properties of the melting layer as a function of frequency, LP RSD ( $\bullet 5$ $\mathrm{mm} / \mathrm{h}, 12.07 \mathrm{~mm} / \mathrm{h}$, Kharadly and Owen, 1988). 


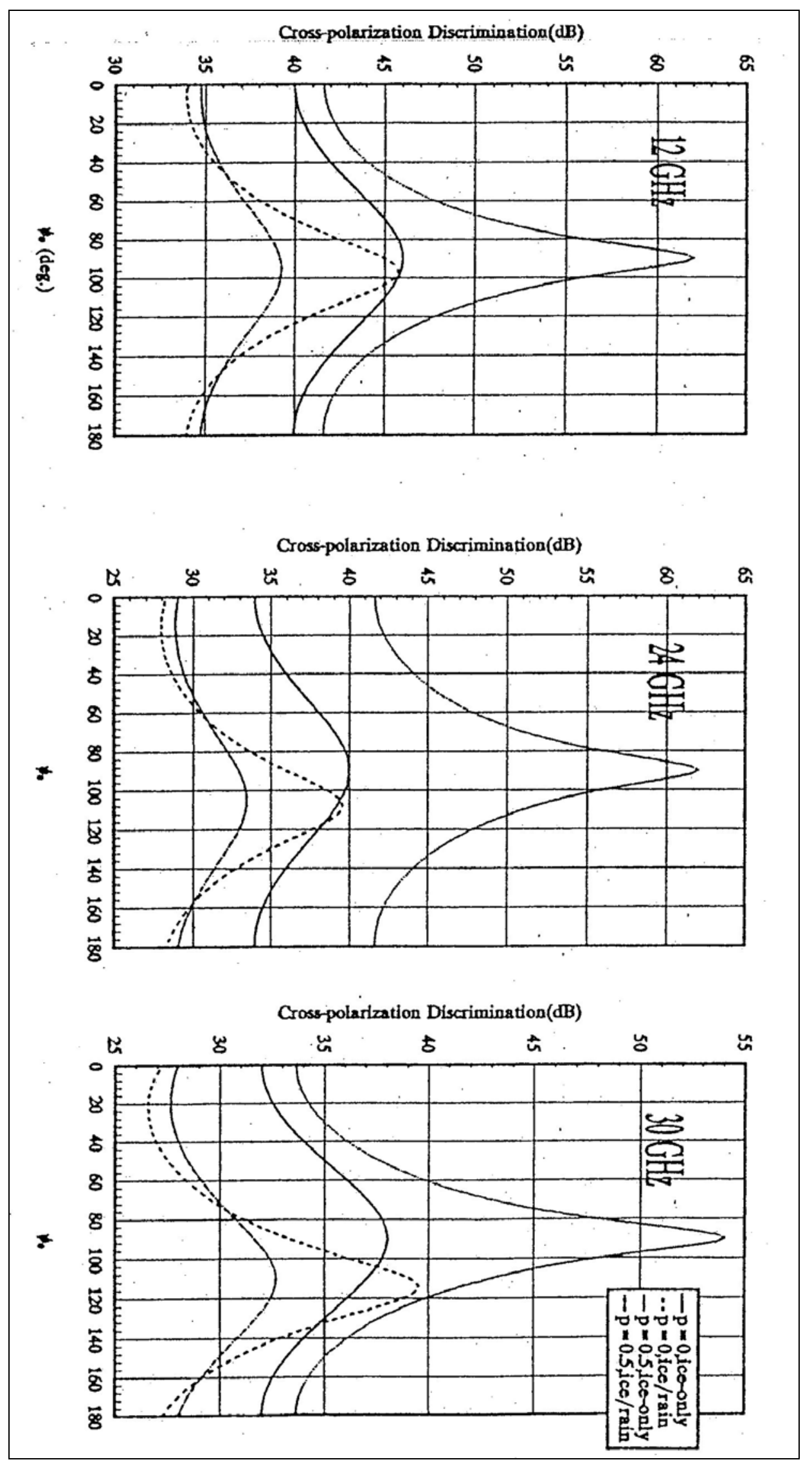

Figure 8. XPD due to a $1-\mathrm{km}$ thick ice slab combined with a $10 \mathrm{~mm} / \mathrm{h}$ rainfall rate along a satellite-to-earth path of $\beta=45^{0}$ versus $\psi_{0}$ of needle-shaped ice crystals for a RHCP transmission. Ice cloud parameters: $H_{c}=1 \mathrm{~km},\langle V\rangle=10^{-7}$ $\mathrm{m}^{3} / \mathrm{m}^{3}, e^{p}=e^{n}=0.8, \chi_{0}=0^{0}, \sigma_{\chi}=0^{0}, \sigma_{\psi}=0^{0}$. Rain parameters: path length $=3 \mathrm{~km}$, MMP RSD, P-P drops at $0^{0} \mathrm{C}$, uniform rain-rate spatial distribution, $\theta_{0}=0^{0}, \sigma_{\theta}=12^{0}$ 

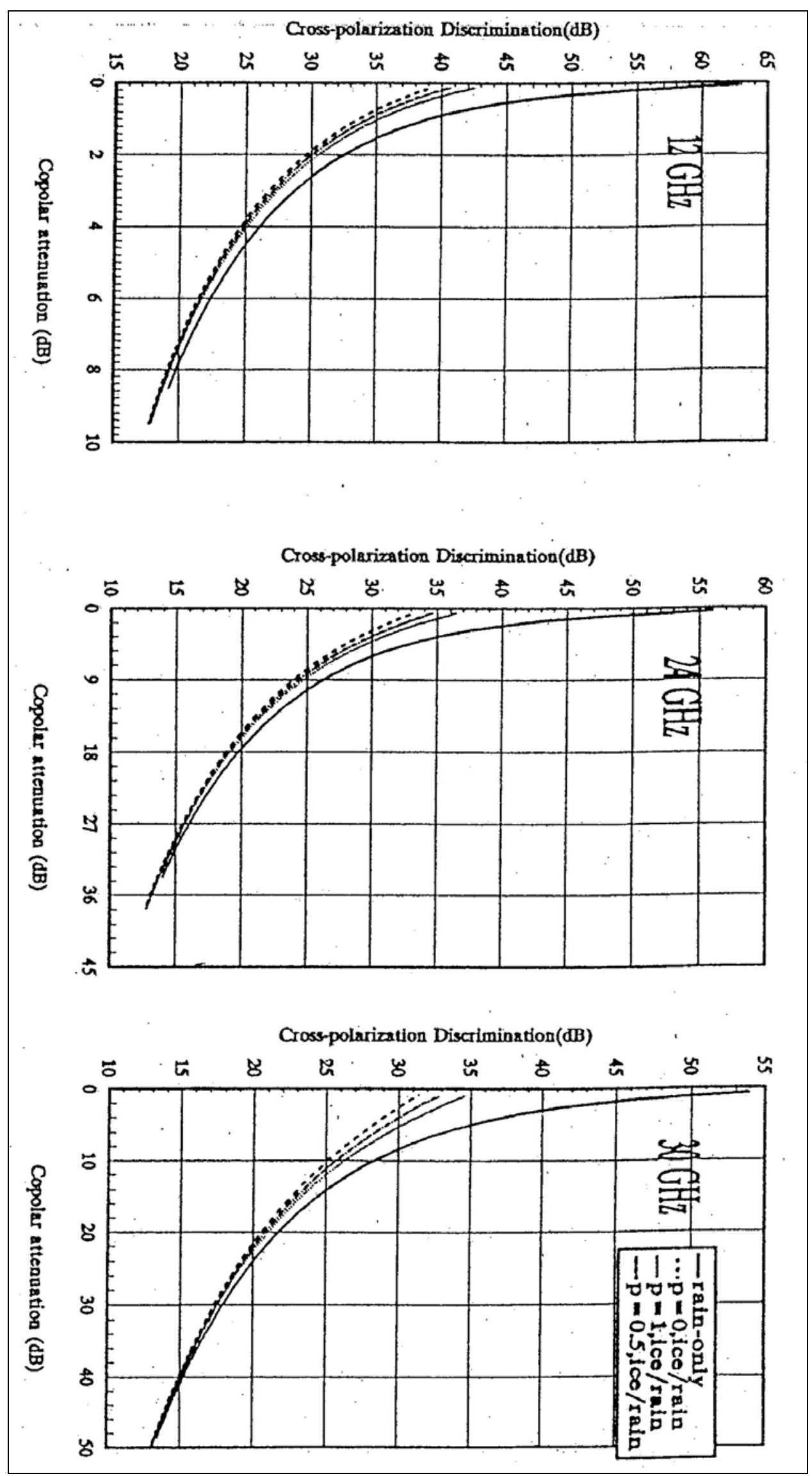

Figure 9. XPD due to a mixed ice/rain medium along a satellite-to-earth path of $\beta=45^{0}$ versus $\alpha$ for a RHCP transmission. Ice cloud parameters: $H_{c}=1 \mathrm{~km},\left\langle V>=10^{-7} \mathrm{~m}^{3} / \mathrm{m}^{3}, e^{p}=e^{n}=0.8, \chi_{0}=\psi_{0}=0^{0}, \sigma_{\chi}=0^{0}, \sigma_{\psi}=0^{0}\right.$. Rain parameters: path length $=3 \mathrm{~km}, \mathrm{MMP} \mathrm{RSD}, \mathrm{P}-\mathrm{P}$ drops at $0^{0} \mathrm{C}$, uniform rain-rate spatial distribution, $\theta_{0}=0^{0}$, $\sigma_{\theta}=12^{0}$ 


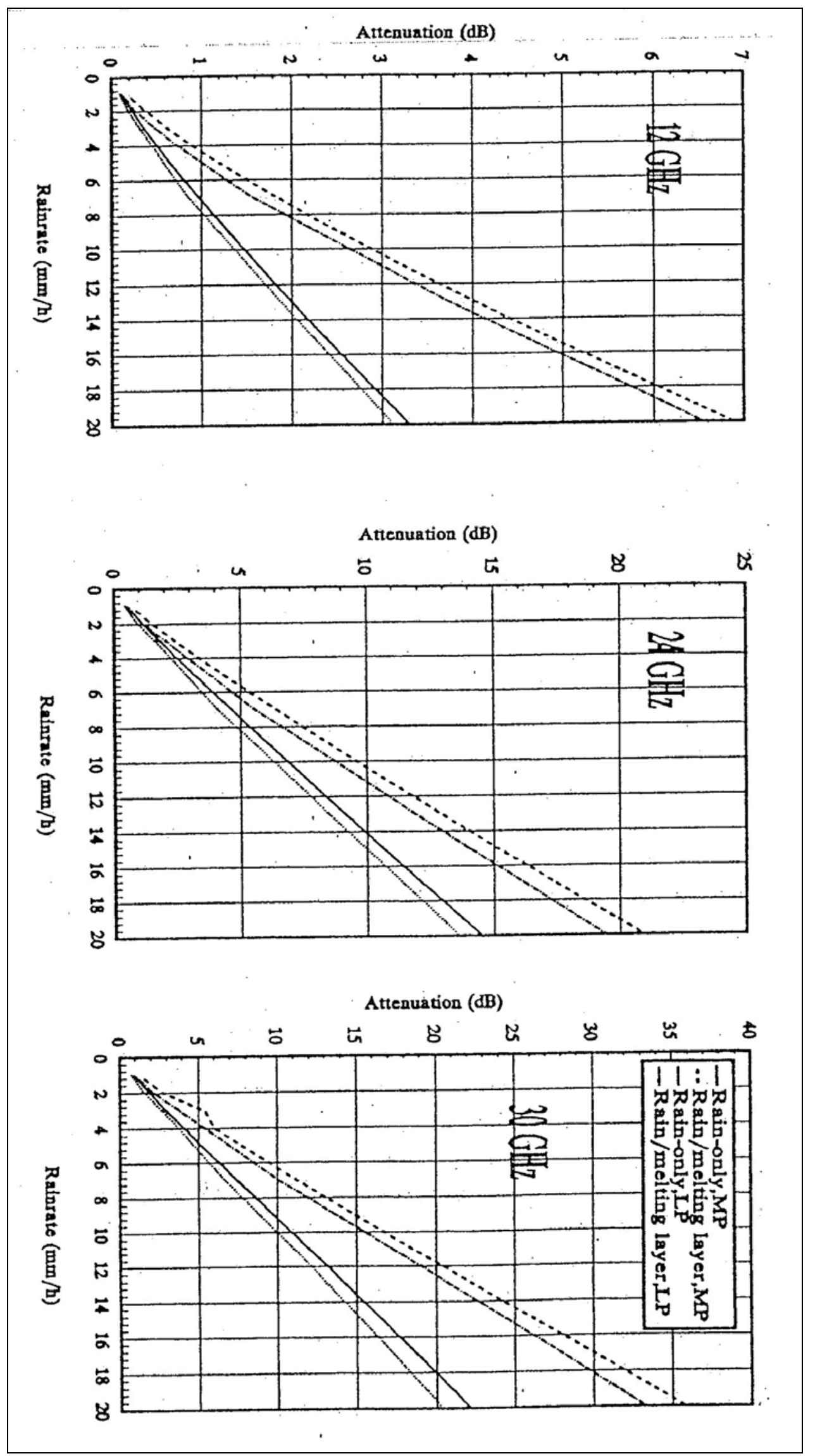

Figure 10. Rain and rain/melting layer total excess attenuation along a satellite-to-earth path of $\beta=45^{\circ}$ versus rainfall rate for a RHCP transmission. Melting layer parameters: path length $=0.5 \mathrm{~km}$, lapse rate of temperature $=6^{0} \mathrm{C}$. Rain parameters: path length $=3 \mathrm{~km}, \mathrm{MP} \mathrm{RSD}, \mathrm{P}-\mathrm{P}$ drops at $0^{0} \mathrm{C}$ shapes, uniform rain-rate spatial distribution, $\theta_{0}=0^{0}, \sigma_{\theta}=12^{0}$ 


\section{Conclusions}

A general PSP has been developed to evaluate the deleterious effects of the whole spectrum of precipitation in the lower part of the atmosphere that may impede the reliability of satellite-earth communications systems. An important aspect of the work reported in this paper is the implementation of the GMT for the EM scattering from realistically distorted raindrops at frequencies which have not been hitherto considered by any other numerical EM computational technique including the point-matching (Morrison and Cross, 1974), (Oguchi, 1977), perturbation (Oguchi, 1964) and (Li, et al., 1995), T-matrix (Warner and Hizal, 1976), integral-equation (Holt, Uzunoglu, and Evans, 1978) and the finite element method (Ajase and Sadiku, 1995). The use of the GMT not only extends the solution to encompass frequencies well above $100 \mathrm{GHz}$, but also takes a step forward in improving the accuracy, particularly of $\Delta \phi$ above $30 \mathrm{GHz}$. To our knowledge, results for $\alpha, \Delta \alpha, \Delta \phi$ and XPD for the rain, melting layer as well as the mixed ice/rain and melting layer/rain media are presented here for the first time.

\section{References}

AJASE S.O. and SADIKU N.O. 1995. Computation of attenuation, phase rotation, and cross polarization of radio waves due to rainfall in tropical regions, IEEE Trans. Antennas Propagat., AP-43: 1-5.

AJAYA G.O. and OLSEN R.L. 1985. Modeling of a tropical raindrop size distribution for microwave and millimeter wave application, Radio Sci., 21: 193-202.

ALLNUT J.E., 1989. Satellite-to-Ground Radio Wave Propagation.: Peter Peregrinus Ltd.London, U.K

AL-RIZZO H.M. and TRANQUILLA J.M. 1997. Application of the generalized multipole technique (GMT) to high-frequency electromagnetic scattering from perfectly conducting and dielectric bodies of revolution, J. Comput. Phys., 136: 1-18.

AL-RIZZO H.M., AL-HAFID H.T., and TRANQUILLA J.M. 2000. Electromagnetic modeling of the propagation characteristics of satellite communications through composite precipitation media, part I: mathematical formulation,. SQUJ. Scientific Res. Science and Tech, 5: 47-54.

DE WOLF D.A. and ZWIESLER A.J. 1996. Rayleigh-Mie approximation for line-of-sight propagation through rain at 5-90 GHz, IEEE Trans. Antennas Propagat., AP-44: 273-279.

DISSANAYAKE A.W. and MCEWAN N.J. 1978. Radar and attenuation properties of rain and bright band, IEE Conf. Publ. 169: 125-129.

EKPENYONG B.E. and SRIVASTAVA R.C. 1970. (February), Radar characteristics of the melting layer- A theoretical study, Tech. Rep. No. 16, Dept. of the Geophys. Sci., The University of Chicago and Illinois Inst. of Techn., Dept. of Elect. Eng., Chicago, Illinois.

EVANS B.G. and HOLT A.R. May 1980. A review of theoretical prediction techniques of transmission parameters for slant-path, earth-space communications, NATO AGARD Conf. On Propagation Effects in Space-Earth paths, London.

FANG D.J. and LEE F.J. 1978. Tabulation of raindrop induced forward and backward scattering amplitudes, Comsat. Tech. Rev., 8: $455-486$.

HOLT A.R., UZUNOGLU N.K., and EVANS B.G. 1978. An integral equation solution to the scattering of electromagnetic radiation by dielectric spheroids and ellipsoids, IEEE Trans. Antennas Propagat., AP-26: 706-712.

KHARADLY M. M. Z. and OWEN N. 1988. (August), Microwave propagation through the melting layer at grazing angles of incidence, IEEE Trans. Antennas Propagat., AP-36: 1106-1113.

KLAASSEN W., 1988 (December). Radar observations and simulation of the melting layer of precipitation, J. Atmos. Sci., 45: 3741-3753.

LAWS J.O. and PARSONS D.A. 1948. The relation of raindrop size to intensity, J. Meteorol., 5: 165-166.

LI LE-WEI, PANG-SHYAN KOOI, MOOK-SENG LEONG, TAT-SOON YEO, and MIN-ZHAN GAO, 1995. Microwave attenuation by realistically distorted raindrops: Part I- Theory, IEEE Trans. Antennas Propagat., AP-43: 811-821.

MORRISON J.A. and. CROSS M.J. 1974. Scattering of a plane electromagnetic wave by axisymmetric raindrops, Bell Syst. Tech. J., 53: 955-1019.

OGUCHI T., 1964. Attenuation of electromagnetic waves due to rain with distorted raindrops (Part II), J. Radio Res. Labs., 11: 1944.

OGUCHI T., 1977. Scattering properties of Pruppacher-and-Pitter form raindrops and cross polarization due to rain : calculations at 11, 13, 19.3 and $34.8 \mathrm{GHz}$, Radio Sci., 12: 41-51.

OLSEN R.L., ROGERS D.V., and HODGE D.B. 1978. The $\mathrm{aR}^{\mathrm{b}}$ relation in the calculation of rain attenuation, IEEE Trans. Antennas Propagat., AP-26: 318-329.

PERSINGER R.P., STUTZMAN W.L., CASTLE R.E. and BOSTIAN C.W. 1980. Millimeter wave attenuation prediction using a piecewise uniform rain rate model, IEEE Trans. Antennas Propagat., AP-28: 149-153.

PRUPPACHER R. and. PITTER R.L. 1971. A semi-empirical determination of the shape of cloud and rain drops, J. Atmos. Sci., 28: 86-94.

SEOW YONG-LEE, LE-WEI LI, MOOK-SENG LEONG PANG-SHYAN KOOI, and TAT-SOON YEO, 1998. An efficient TCS formula for rainfall microwave attenuation: T-matrix approach and 3-D fitting for oblate spheroidal raindrops, IEEE Trans. Antennas Propagat., AP-46: 1176-1181.

STUTZMAN W.L. and DISHMAN W.K. 1982. A simple model for the estimation of rain-induced attenuation along earth-space paths at millimeter wavelengths, Radio Sci., 17: 1465-476. 


\section{MODELING THE PROPAGATION OF SATELLITE COMMUNICATIONS}

TRANQUILLA J. M. and AL-RIZZO H. M. 1994. (February), Investigation of GPS precise relative positioning during periods of ice clouds and snowfall precipitation, IEEE Trans. Antennas Propagat., AP- 42: 157-165.

WARNER C. and HIZAL A., 1976. Scattering and depolarization of microwaves by spheroidal raindrops, Radio Sci., 11: 921-930.

Received 1 December 1999

Accepted 19 June 2000 